\title{
聚酰亚胺膜的分子结构与透气性 能之间关系的研究
}

李说生丁孟贤*张 动 徐纪平

（中国科学院长春应用化学研究所, 长春 130022)

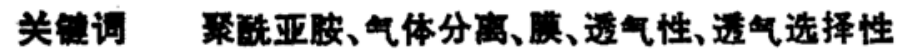

理想的气体分离膜材料应该同时具有高透气性和高透气选择性、高机械强度、优良的热和 化学稳定性以及良好的成膜加工性能。在商业高分子材料中, 透气性好的材料往往透气选择 性较差,而透气选择性好的材料往往透气性差,一般难以找到理想的气体分离膜材料 ${ }^{[1,2]}$. 为了 发展好的气体分离膜材料, 我们系统地研究了聚酰亚胺的分子结构与气体分离性能之间的关 系. 在前文 ${ }^{[3]}$ 的基础上,本文报道了二苯酮二酐 (BTDA) 型和二苯硫醚二䣶 (TDPA) 型取 代聚酰亚胺的气体分离性能, 这类聚酰亚胺具有很高的透气性和透气选择性, 是比较好的气体 分离膜材料.

\section{1 实 验 部 分}

二苯酮二酐 (B'DA) 为日本 Daciel 公司产品. 二苯硫醚二酐 (TDPA) 由本实验室合 成, 用前经真空升华精制. 3, 3'-二甲基二苯甲烷二胺（DMMDA，2，2', 3, 3'-四甲基二苯甲 烷二胺 (TMMDA1) 和 2,2',5,5'-四甲基二苯甲烷二胺（TMMDA2）均由本实验室合成, 用 前均经过重结晶精制. $\mathrm{N}, \mathrm{N}$-二甲基乙酰胺 (DMAc) 为上海试剂三厂化学纯试剂, 用前经 4A 分子篲干蜗,蒸馏精制. 各种气体的纯度均大于 $99.5 \%$ 。聚酰亚胺的合成和膜的制备按文<smiles>Cc1cc(Cc2ccc(N)c(C)c2)ccc1N</smiles><smiles></smiles>

ITMMDAI<smiles>Cc1cc(Cc2cc(C)c(N)cc2C)c(C)cc1N</smiles><smiles>O=C(c1ccc2c(c1)C(=O)OC2=O)c1ccc2oc(=O)oc2c1</smiles>

BTDA<smiles>O=C1OC(=O)c2cc(Sc3ccc4c(c3)C(=O)OC4=O)ccc21</smiles>

图 1 聚酰亚胺膜的分子结构

1993-02-19 收稿, 1993-06-18 收修改秒。

- 通讯联系人. 
献 [4]进行, 膜厚度为 $35-40 \mu \mathrm{m}$. 聚酰亚胺膜的表征按文献 [3]进行.

\section{2 结果与讨论}

\section{1 聚酰亚胺膜的制备与表征}

本文研究的聚酰亚胺膜的分子结构如图 1, 除 BTDA-DMMDA 外, 其它聚酰亚胺均是未 见报道的新聚合物. 我们发现当采用化学酰亚胺化法合成本文研究的取代聚酰亚胺时, 它们 可溶于二甲基甲酰胺、二甲基乙酰胺和 N-甲基吡咯烷酮等极性非质子溶剂, 这对于制备具有 实用意义的不对称中空纤维是非常有利的, 所以本文采用低温溶液缩聚-化学酰亚胺化法制备 聚酰亚胺膜。

表 1 是取代聚酰亚胺膜的有关物理性质. 由表 1 可知,在聚酰亚胺的分子中引入甲基后, 聚酰亚胺的 $T_{\mathrm{g}}$ 升高, 甲基越多, $T_{\mathrm{g}}$ 越高, 这是因为苯环中的甲基限制了 $\mathrm{N}-\mathrm{Ar}$ 键和 $\mathrm{C}-\mathrm{Ar}$ 的旋转. $T_{\mathrm{g}}$ 的高低可大致反映出聚合物分子链段活动性的相对大小, 所以两类聚酰亚胺的链 没活动性大小顺序均为

MDA 型 $>$ DMMDA 型 $>$ TMMDA 1 型 $\sim$ TMMDA2 型.

表 1 所列的自由体积 $\left(V_{\mathrm{f}}\right)$ 是按基团求和法 ${ }^{[5,6]}$ 计算得到的. 在聚酰亚胺的分子中引人 甲基后,聚酰亚胺的自由体积增大,两类聚酰亚胺的自由体积大小顺序均为

$$
\text { MDA 型 }<\text { DMMDA 型 }<\text { TMMDA1 型 TMMDA2 型. }
$$

表 1 聚酰亚胺膜的有关物理性质

\begin{tabular}{l|c|c|c}
\hline \multicolumn{1}{c|}{ 聚酰亚胺 } & $T_{\mathrm{k}}\left({ }^{\circ} \mathrm{C}\right)$ & $\rho\left(\mathrm{g} / \mathrm{cm}^{3}\right)$ & $V_{\mathrm{f}}\left(\mathrm{cm}^{3} / \mathrm{g}\right)$ \\
\hline BTDA-MDA & 280 & 1.346 & 0.084 \\
BTDA-DMMDA & 288 & 1.304 & 0.094 \\
BTDA-TMMDA1 & 307 & 1.248 & 0.098 \\
BTDA-TMMDA2 & 308 & 1.249 & 0.097 \\
TDPA-MDA & 265 & 1.343 & 0.091 \\
TDPA-DMMDA & 273 & 1.340 & 0.094 \\
TDPA-TMMDA1 & 299 & 1.300 & 0.096 \\
TDPA-TMMDA2 & 296 & 1.299 & 0.097 \\
\hline
\end{tabular}

表 2 聚酰亚胺的透气系数 ${ }^{(n)}$

\begin{tabular}{|c|c|c|c|c|c|c|}
\hline \multirow{2}{*}{ 聚酰亚胺 } & \multicolumn{3}{|c|}{$30^{\circ} \mathrm{C}$} & \multicolumn{3}{|c|}{$100^{\circ} \mathrm{C}$} \\
\hline & $P_{\mathrm{H}_{2}}$ & $P_{\mathrm{O}_{2}}$ & $P_{\mathrm{N}_{2}}$ & $P_{\mathrm{H}_{2}}$ & $P_{\mathrm{O}_{2}}$ & $P_{\mathrm{N}_{2}}$ \\
\hline $\mathrm{BTDA}-\mathrm{MDA}^{[3]}$ & 4.03 & 0.211 & 0.0196 & 11.4 & 0.716 & 0.110 \\
\hline BTDA-DMMDA & 8.25 & 0.328 & 0.0300 & 20.8 & 0.161 & 0.161 \\
\hline BTDA-TMMDA1 & 13.1 & 0.524 & 0.0553 & 32.6 & 1.49 & 0.276 \\
\hline BTDA-TMMDA2 & 12.9 & 0.518 & 0.0533 & 32.4 & 1.50 & 0.256 \\
\hline TDPA-MDA ${ }^{[3]}$ & 5.43 & 0.246 & 0.0270 & 14.8 & 0.827 & 0.152 \\
\hline TDPA-DMMDA & 9.15 & 0.350 & 0.0377 & 21.3 & 1.01 & 0.181 \\
\hline TDPA-TMMDA1 & 14.4 & 0.610 & 0.0649 & 32.4 & 1.71 & 0.297 \\
\hline TDPA-TMMDA2 & 14.9 & 0.618 & 0.0673 & 32.2 & 1.77 & 0.310 \\
\hline
\end{tabular}

a) 倵压力: 0.7 MPa, 遥气系数的单位: barrar. 


\section{2 聚酰亚胺的透气性能}

气体在致密高分子膜中的透过是按照“溶解-扩散”机制进行的,透气系数的大小与高分子 的链段活动性和自由体积有关.一般来讲, 具有较大自由体积和较小链段活动性的高分子膜 材料具有较大的透气性和较大的透气选择性 ${ }^{[7]}$. 三种 BTDA 型取代聚酰亚胺的自由体积均 比 BTDA-MDA 的大得多, 而链段活动性比 BTDA-MDA 的小, 所以，与 BTDA-MDA 相 比, 它们具有较高的透气性和透气选择性 (见表 1 和 2); TDPA 型聚酰亚胺的情况与此类 似.由于链段活动性比 BTDA 型聚酰亚胺的小,因此, TDPA 型聚酰亚胺的透气性比 BTDA 型聚酰亚胺好一些,透气选择性比 BTDA 型聚酰亚胺差一些.

表 3 聚酰亚胺的透气选择系数

\begin{tabular}{|c|c|c|c|c|}
\hline \multirow{2}{*}{ 聚㱡亚桉 } & \multicolumn{2}{|c|}{$30^{\circ} \mathrm{C}$} & \multicolumn{2}{|c|}{$100^{\circ} \mathrm{C}$} \\
\hline & $\alpha_{\mathrm{H}_{2} / \mathrm{N}_{2}}$ & $\boldsymbol{\alpha}_{\mathrm{O}_{2} / \mathrm{N}_{2}}$ & $\alpha_{\mathrm{H}_{2} / \mathrm{N}_{2}}$ & $\alpha_{\mathrm{O}_{2} / \mathrm{N}_{2}}$ \\
\hline BTDA-MDA ${ }^{[3]}$ & 206 & 10.8 & 104 & 6.5 \\
\hline BTDA-DMMDA & 275 & 9.6 & 129 & 6.2 \\
\hline BTDA-TMMDAI & 237 & 9.7 & 118 & 6.0 \\
\hline BTDA-TMMDA2 & 242 & 9.4 & 121 & 5.9 \\
\hline TDPA-MDA ${ }^{[3]}$ & 201 & 9.3 & 97.3 & 5.6 \\
\hline TDPA-DMMDA & 243 & 9.3 & 118 & 5.9 \\
\hline TDPA-TMMDAI & 222 & 9.3 & 109 & 5.8 \\
\hline TDPA-TMMDA2 & 221 & 9.2 & 107 & 5.7 \\
\hline
\end{tabular}

80 年代中期, 日本的宇部兴产公司开发了宇部气体膜分离器 (Ube Gas Separatore), 人 们普迫认为 Ube Gas Separatore 是迄今为止世界上最先进的 $\mathrm{H}_{2}$ 回收用气体膜分离器. 宇 部采用的膜材料是联苯型聚酰亚胺,室温下，其透 $\mathrm{H}_{2}$ 系数为 $566.610 \mathrm{~Pa}$ 左右, $\mathrm{H}_{2} / \mathrm{N}_{2}$ 分离 系数为 165. 从表 2 和表 3 可知, $30^{\circ} \mathrm{C}$ 时, 三种带有取代基的 BTDA 型聚酰亚胺 BTDADMMDA, BTDA-TMMDA1 和 BTDA-TMMDA2 的透 $\mathrm{H}_{2}$ 系数分别比宇部膜材料高 60 , 160 和 $150 \%$ 左右, $\mathrm{H}_{2} / \mathrm{N}_{2}$ 分离系数分别比宇部膜材料高 65,45 和 $47 \%$ 左右, 三种带有取代 基的 TDPA 型聚酰亚胺 TDPA-DMMDA, TDPA-TMMDA1 和 TDPA-TMMDA2 的透 $\mathrm{H}_{2}$ 系数分别比宇部膜材料高 80,180 和 $190 \%$ 左右, $\mathrm{H}_{2} / \mathrm{N}_{2}$ 分离系数分别比宇部膜材料离 45,35 和 $35 \%$ 左右, 而且这些取代聚酰亚胺的溶解性能均好于宇部膜材料, 是比较好的 $\mathrm{H}_{2}$ 回 收用气体分离膜材料.

\section{参考献}

[1] Koros, W. J., Fleming, G. R. et al., Prog. Polym. Sci., 1988, 13: 339.

[2]李说生、丁孟贤、徐纪平, 高分子通讯, $1991,(3): 138$.

[3] 李说生、丁孟贤、徐纪平, 应用化学, $1992,9(3): 87$.

[4] Kim, T. H., Koros,W. J., O'brien, G. R., J. Membr. Sci., 1989, 37: 45.

[5] Lee, W. M., Polym. Eng.Sci., 1980, 20: 65.

[6] Vankrvelen, D. W., Hoftyzer, P. J., Properties of Polymers,2nd ed., Chap. 4, Elsevier, New York, 1976.

[7] Hanaka, K., Kita, H. et al., 膜 (Membrane), 1986, $11: 48$. 